\title{
Angular momentum transfer in optically induced photonic lattices
}

\author{
Milan S. Petrović, ${ }^{1}$ Dragana M. Jović, ${ }^{1}$ Milivoj R. Belić, ${ }^{1,2}$ and Slobodan Prvanović ${ }^{1}$ \\ ${ }^{1}$ Institute of Physics, P.O. Box 57, 11001 Belgrade, Serbia \\ ${ }^{2}$ Texas A\&M University at Qatar, P.O. Box 5825, Doha, Qatar
}

(Received 26 September 2006; published 21 August 2007)

\begin{abstract}
The transfer of orbital angular momentum from vortex beams to optically induced photonic lattices is demonstrated. It is found that the sum of the angular momenta of interacting incoherent counterpropagating (CP) beams is not conserved, whereas their difference is. The sum of angular momenta of copropagating (CO) interacting beams is strictly conserved. It is also found that the transfer of angular momentum in $\mathrm{CP}$ interacting beams is minimal, amounting to a few percent, whereas the transfer in $\mathrm{CO}$ interacting beams is substantial, amounting to tens of percent. In fixed lattices, for both $\mathrm{CP}$ and $\mathrm{CO}$ beams, angular momentum is never conserved.
\end{abstract}

DOI: 10.1103/PhysRevA.76.023820

PACS number(s): 42.65.Tg, 42.65.Sf

\section{INTRODUCTION}

A surge of interest in light displaying angular momentum (AM) while propagating in nonlinear (NL) media has been noted recently $[1,2]$. It was spurred by the interesting applications of beams carrying optical AM, such as for driving micromachines [3], optical tweezers [4], or quantum cryptography [5]. It has also been a controversial topic, in the sense that AM turned out not to be conserved in certain situations involving the propagation of multicomponent light fields in NL media; see, for example, the contrasting points of view on the spiraling of solitons in photorefractive (PR) crystals [6], or on the spontaneous parametric downconversion in barium borate crystals $[5,7]$.

We discuss here a few additional examples where the total $\mathrm{AM}$ is not a conserved quantity. The motivation comes from an interesting paper [8] by Chen et al., in which the interaction between propagating laser beams and optically induced photonic lattices in PR crystals [9] was investigated experimentally. At one point the authors posed an intriguing question: Can the angular momentum carried by a vortex beam be transferred to a nonlinear lattice? In this paper, we answer the question in the affirmative and analyze various possibilities for the momentum transfer. In the analysis it transpired that the behavior of interacting copropagating $(\mathrm{CO})$ and counterpropgating $(\mathrm{CP})$ beams is fundamentally different: the sum of the AMs of $\mathrm{CO}$ beams is conserved, whereas in the $\mathrm{CP}$ case the conserved quantity is the difference of AMs. The conservation of AM is prevented if we artificially fix the lattices. The fixed lattice approximation can be justified if the maximum lattice intensity is much greater than the maximum beam intensity, which is often the case in experiments.

We utilize a model that describes the NL propagation of mutually incoherent CP beams and optically induced photonic lattices in PR crystals, and allow for the existence of defects in the lattice [10]. Although the experiment [8] is done in a $\mathrm{CO}$ geometry, we extend the analysis to the more general CP geometry, so as to obtain more general answers. Our results can be extended to other interesting systems, such as lattices and beams in Bose-Einstein condensates [11], microwave solitons in magnetic films [12], and even confined beam structures in left-handed metamaterials [13].
We find that the transfer of orbital AM is minimal in interacting CP lattices, while it can be substantial in fixed lattices. Denoting by $F$ and $B$ the envelopes of the forward and backward propagating beams, and by $G_{f}$ and $G_{b}$ the envelopes of the lattice beams, we establish that the total AM-meaning the sum $L z(F)+L z\left(G_{f}\right)+L z(B)$ $+L z\left(G_{b}\right)$ of all momenta along the propagation $z$ axis-is not conserved in the case of $\mathrm{CP}$ interacting beams. More precisely, the difference of the AMs of $\mathrm{CP}$ beams $L z(F)$ $+L z\left(G_{f}\right)-L z(B)-L z\left(G_{b}\right)$ is conserved, whereas their sum is not. The sum is conserved only in the pure CO case. In addition, in the fixed lattices there is always a considerable loss of AM. The standard definition for the (normalized) $z$ component of the orbital AM is adopted, $L z(F)$ $=-(i / 2) \iint d x d y F^{*}(x, y)\left(x \partial_{y}-y \partial_{x}\right) F(x, y)+$ c.c., and similarly for other components [14].

\section{NUMERICAL RESULTS AND DISCUSSION}

We utilize a well-known $[15,16]$ local model for the generation of a space charge field in PR crystals, and consider beam propagation in the paraxial approximation. The following model equations for the interacting incoherent beams in the computational domain are used:

$$
\begin{gathered}
i \partial_{z} F=-\Delta F+\Gamma E F, \quad-i \partial_{z} B=-\Delta B+\Gamma E B, \\
i \partial_{z} G_{f}=-\Delta G_{f}+\Gamma E G_{f}, \quad-i \partial_{z} G_{b}=-\Delta G_{b}+\Gamma E G_{b}, \\
\tau \partial_{t} E+E=-\frac{I+I_{g}}{1+I+I_{g}},
\end{gathered}
$$

where $\Delta$ is the transverse Laplacian and $\Gamma$ the beam coupling constant. $E$ is the space charge field built in the crystal, normalized to the applied external field, and $I=|F|^{2}+|B|^{2}$ and $I_{g}=\left|G_{f}\right|^{2}+\left|G_{b}\right|^{2}$ are the corresponding beam intensities, measured in units of the dark or background intensity $I_{d}$. $\tau$ is the relaxation time of the crystal. Time derivatives do not appear in Eqs. (1) because the fast optical fields are slaved to the slow $E$ field, and spatial derivatives are absent from Eq. (2) 
because a local isotropic interaction is assumed. These assumptions are reasonable for slow PR media. The same model describes the pure $\mathrm{CO}$ case, when $B$ and $G_{b}$ fields are absent. Similarly, the case of fixed lattices, an approximation often used, is obtained by eliminating Eqs. (1b) and incorporating the fixed lattice intensity distribution into Eq. (2). Throughout, we are concerned only with the steady state.

The propagation equations (1) are solved numerically, concurrently with the temporal equation (2) for the space charge field, in the manner described in Ref. [15] and for typical values of parameters mostly used in experiments. The numerical procedure here is modified, in that the CP lattice beams are turned on first, to allow lattices to establish themselves, and then the forward and backward beams are turned on. The forward and backward lattices are matched in position, spacing, and intensity, and build a stable structure in the crystal. Head-on CP Gaussian beam arrays are used for the lattices, with a finite trigonal/hexagonal arrangement of beams, and with or without a central defect. Head-on CP vortices with opposite topological charges $( \pm 1)$ are launched in the center of each lattice. In such a system with preserved rotational symmetry of the model equations, the difference of AMs of CP beams is conserved.

A restriction in the form of a two-dimensional periodic potential of a fixed photonic lattice breaks the rotational symmetry of the system, leading to AM nonconservation. In the case of CP beams in a fixed lattice, Eq. (1b) is eliminated, while the fixed lattice intensity distribution is incorporated into Eq. (2), so the symmetry of the remaining model equations is broken, and the sum or difference of AMs is not conserved. When one vortex beam and one lattice beam propagate in the same direction ( $\mathrm{CO}$ interacting beams), beams $B$ and $G_{b}$ are zero, the rotational symmetry of such reduced model equations is unbroken, and the sum of AMs is conserved. Further reduction of the previous model equations (also the CO case, but with a fixed lattice), leads to their symmetry breaking, and there is no conservation of AM. Typical examples are depicted in Figs. 1-4. All the figures are produced in the same setup and scale, and only with minor differences in parameters, as noted along the way.

Figure 1 depicts the situation with interacting $\mathrm{CP}$ lattices and beams. As a result of the interaction, the sum of all AMs remains nearly constant. However, when the difference is calculated, it is found exactly constant. The presence of a defect in the center of the lattice (the second column in Fig. 1) does not change our findings appreciably, the reason being that the $\mathrm{CP}$ lattice beams quickly regenerate the missing central beam.

The situation changes drastically for $\mathrm{CP}$ beams in a fixed lattice (Fig. 2), where one finds a much larger AM drop than in the case of interacting CP lattices. The fixed lattice is "heavy" and it does not interact with the CP vortices, so the transfer of AM is then precluded, and the law of AM conservation is no longer valid. The propagation of $\mathrm{CP}$ vortices proceeds in a discrete periodic potential, in this case of $C_{6 v}$ symmetry, and the $\mathrm{O}(2)$ symmetry is gone. AM is no longer a good "quantum number." A new conserved quantity must be introduced, the Bloch or pseudo-AM [17]. The situation is akin to the propagation of $\mathrm{CP}$ vortices in photonic crystals

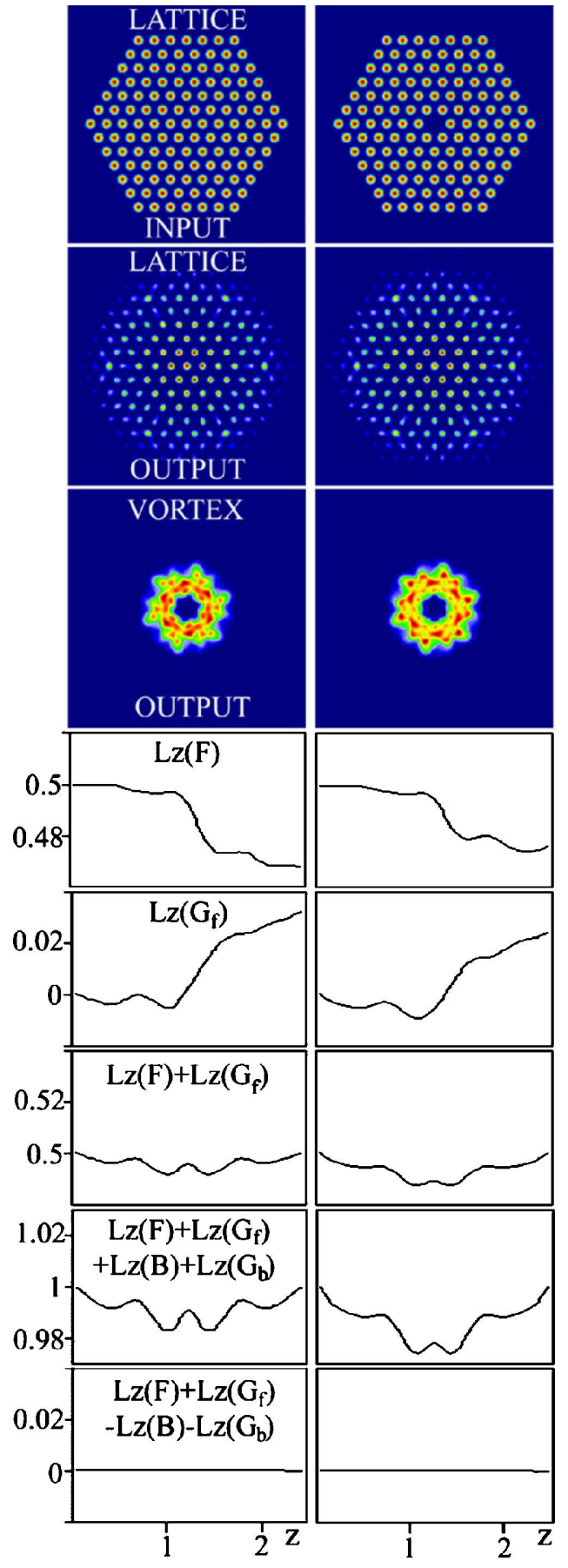

FIG. 1. (Color online) Transfer of AM for interacting CP lattices and beams. The first column presents the lattice without defects, the second column presents the lattice with a defect in the center. The first row presents the input forward lattice intensity, the second and third rows show the intensity distributions of the lattice and the vortex at the output. The remaining five rows present various AMs. Parameters: $\Gamma=4, L=2.5 L_{D}=10 \mathrm{~mm}$, lattice spacing $28 \mu \mathrm{m}$, full width at half maximum (FWHM) of input vortices $28 \mu \mathrm{m}$, FWHM of input lattice beams $9 \mu \mathrm{m}$, maximum input intensity $\left|F_{0}\right|^{2}=\left|B_{L}\right|^{2}$ $=5 I_{d}$, and maximum input lattice intensity $\left|G_{f}\right|_{z=0}^{2}=\left|G_{b}\right|_{z=L}^{2}=20 I_{d}$.

with dielectric rods. The presence of a transverse pointsymmetry group changes the propagation behavior considerably [16]. One notes discrete diffraction, with strong pinning 


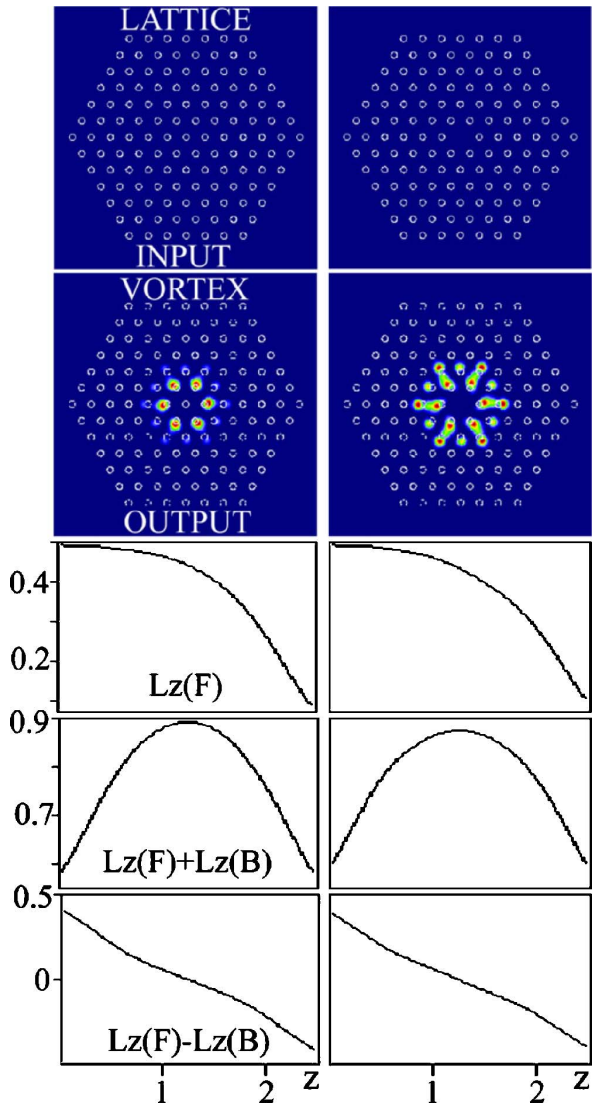

FIG. 2. (Color online) Loss of AM of CP beams in fixed lattices. The layout is as in Fig. 1. The first row depicts the lattices, the second row presents the intensity distribution of the forward vortex within the lattice at its output. The other three rows present different AMs. Parameters are as in Fig. 1, except for the maximum lattice input intensity of $40 I_{d}$, which was chosen in order to keep the same total lattice intensity $I_{g}$ as for Fig. 1.

of vortex filaments to the lattice sites. Neither the sum nor the difference of AM is conserved. The inclusion of a defect in the lattice again does not change our conclusions appreciably.

The behavior noted in the interacting and fixed lattices can be rigorously explained. According to [14], the derivative of the difference of AM along the $z$ axis for the model at hand and for the assumed CP geometry of propagation in the steady state is given by

$$
\begin{gathered}
\frac{\partial L z(F)}{\partial z}+\frac{\partial L z\left(G_{f}\right)}{\partial z}-\frac{\partial L z(B)}{\partial z}-\frac{\partial L z\left(G_{b}\right)}{\partial z} \\
=\int_{0}^{\infty} \rho d \rho \Gamma \int_{0}^{2 \pi} d \varphi E \frac{\partial\left(|F|^{2}+\left|G_{f}\right|^{2}+|B|^{2}+\left|G_{b}\right|^{2}\right)}{\partial \varphi} \\
=\left.\int_{0}^{\infty} \rho d \rho \Gamma\left[\ln \left(1+I+I_{g}\right)-I-I_{g}\right]\right|_{0} ^{2 \pi},
\end{gathered}
$$

where $\rho$ and $\varphi$ are the cylindrical coordinates. The difference of $\mathrm{AM}$ is conserved in the case of interacting CP lattices, because the space charge field $E$ is then an explicit function of $I+I_{g}$, the integration in $\varphi$ is over a perfect derivative,

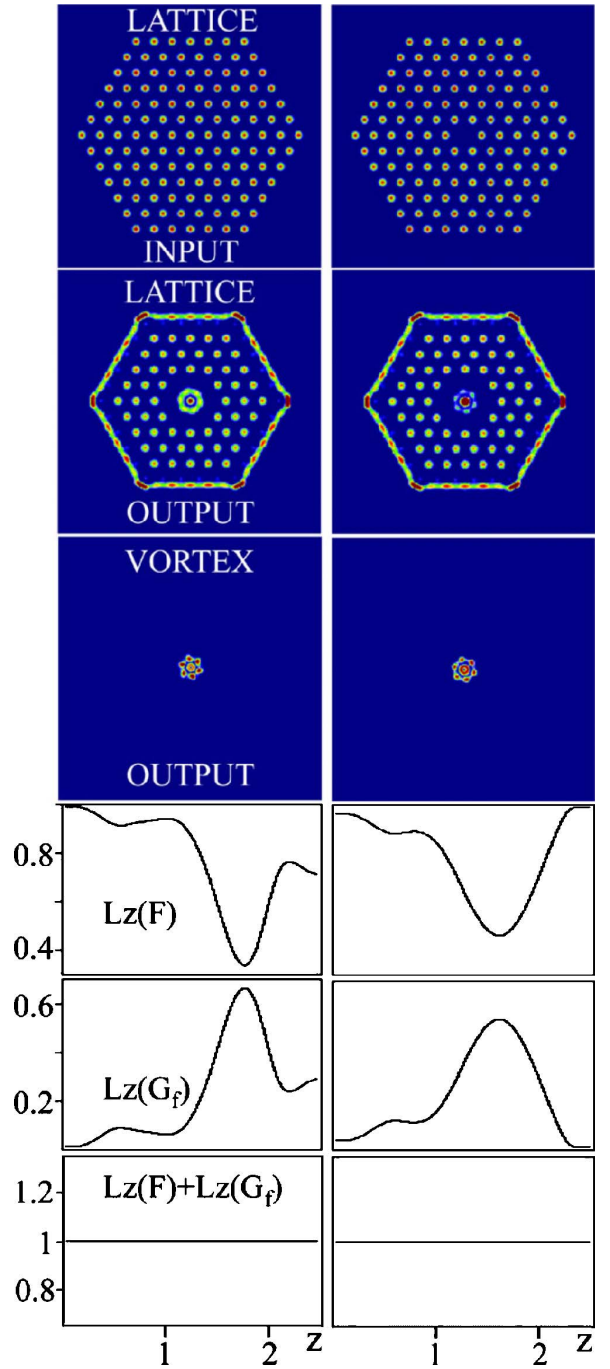

FIG. 3. (Color online) AM transfer in $\mathrm{CO}$ interacting beams. The setup is as in Fig. 1. The only parameter different from those of Fig. 1 is $\Gamma=16$.

and the integral in Eq. (3) is 0. However, for fixed lattices the terms involving $G_{f}$ and $G_{b}$ are absent, while $E$ still contains $I+I_{g}$, and the integration in Eq. (3) is not over a perfect derivative, so the integral does not vanish (Fig. 2); the additional term, which is equivalent to $6 \Gamma \int_{0}^{\infty} \rho d \rho \int_{0}^{\pi / 3}\left(I+I_{g}\right) /\left(1+I+I_{g}\right)\left(\partial I_{g} / \partial \varphi\right) d \varphi$, is not equal to zero [due to the fact that $I(\rho, \varphi, z) \neq I(\rho, \pi / 3-\varphi, z)]$. Therefore, the difference in AM is then not conserved.

The same Eq. (3) leads to the nonconservation of the sum of $\mathrm{AM}$ in the general $\mathrm{CP}$ case, for both the interacting and fixed lattices. The integral then contains the difference of intensities, while $E$ still contains the sum, so it cannot be equal to 0

The CO case offers qualitatively and quantitatively different results from the $\mathrm{CP}$ case. The numerical procedure in the $\mathrm{CO}$ case is considerably simpler, owing to the basic difference between the $\mathrm{CO}$ and $\mathrm{CP}$ processes: the first one is an initial-value problem, whereas the second one is a two-point boundary-value problem. The parameters in both cases are chosen the same, the only difference being the size of the 


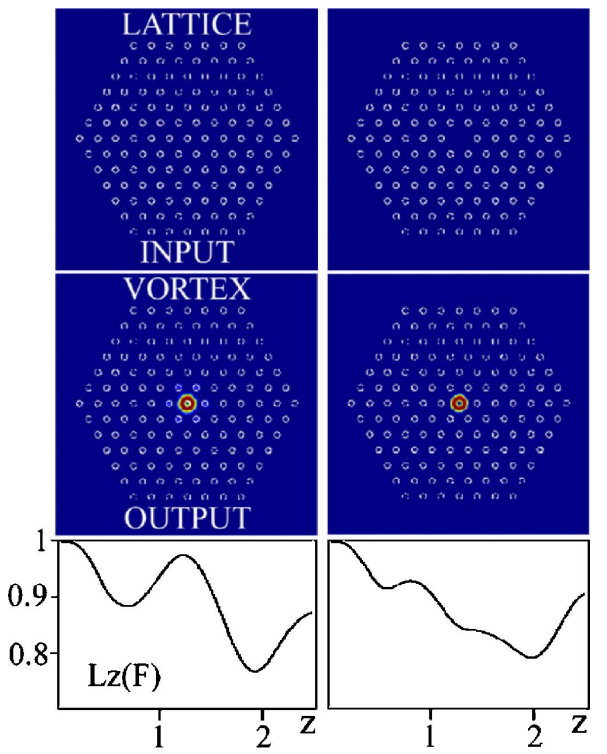

FIG. 4. (Color online) AM loss of a vortex propagating in fixed lattices. The parameters are as in Fig. 3, the setup as in Fig. 2.

coupling parameter $\Gamma$ : the $\mathrm{CO}$ case requires a larger value of $\Gamma$, to build a stable solitonic lattice. Our results are presented in Figs. 3 and 4.

It is seen in Fig. 3 that the transfer of AM is more considerable in the $\mathrm{CO}$ case with interacting beams. The total $\mathrm{AM}$ is conserved. One also notes much stronger finite-size effects at the lattice edge, because of the larger $\Gamma$ coupling. The CO case with a fixed lattice (Fig. 4) mimics the behavior of the $\mathrm{CP}$ case with a fixed lattice. Its $\mathrm{AM}$ is not conserved, and the loss of AM is relatively large. The influence of defects in both cases is presented by the second columns of Figs. 3 and 4. All our numerical results (Figs. 1-4) hold for all (physically reasonable) control parameters.

In the $\mathrm{CO}$ case, a simplified Eq. (3) with the terms $B$ and $G_{b}$ absent, according to the same arguments as above, leads to the conclusions that the total AM is conserved in the case of an interacting lattice (Fig. 3), while AM is not conserved in the case of a fixed lattice (Fig. 4).

\section{CONCLUSIONS}

We stress the essential difference between the $\mathrm{CO}$ and $\mathrm{CP}$ cases. The transfer of AM in CP interacting beams is minimal, while the transfer in $\mathrm{CO}$ interacting beams is substantial. For CO interacting incoherent beams, the sum of the $\mathrm{AM}$ is always constant. However, for $\mathrm{CP}$ interacting incoherent beams, owing to the opposite propagation directions, the difference of AM is constant, but the total AM is not. These conclusions should not be so surprising: they are consistent, for example, with the Manley-Rowe relations for the degenerate two-wave mixing in PR media, in the transmission and reflection geometries. In the case of fixed lattices, for both $\mathrm{CP}$ and $\mathrm{CO}$ beams, the conservation of AM is no longer valid, because this restriction breaks the rotational symmetry of the model equations. Fixed lattices are not an ideal physical approximation, and nonconservation of AM as well as different outputs (comparing to the corresponding cases of interacting lattices) are its consequences. Our results suggest that the difference of AM is conserved in all physical systems where interaction occurs between CP incoherent beams.

\section{ACKNOWLEDGMENTS}

Work at the Institute of Physics is supported by the Ministry of Science Project OI 141031. We are thankful to the IT Center of the Texas A\&M University at Qatar, for allowing the use of the SAQR supercomputing cluster.
[1] L. Allen, S. M. Barnett, and M. J. Padgett, Optical Angular Momentum (Institute of Physics Publishing, Bristol, 2003).

[2] L. Allen, M. J. Padgett, and M. Babiker, Prog. Opt. 39, 291 (1999).

[3] P. Galajda and P. Ormos, Appl. Phys. Lett. 78, 249 (2001).

[4] K. Dholakia, G. Spalding, and M. MacDonald, Phys. World 15, 31 (2002).

[5] A. Mair, A. Vaziri, G. Weihs, and A. Zeilinger, Nature (London) 412, 313 (2001).

[6] M. F. Shih, M. Segev, and G. Salamo, Phys. Rev. Lett. 78, 2551 (1997); V. Tikhonenko, J. Christou, and B. LutherDavies, ibid. 76, 2698 (1996); D. V. Skryabin, J. M. McSloy, and W. J. Firth, Phys. Rev. E 66, 055602(R) (2002); M. R. Belić, D. Vujić, A. Stepken, F. Kaiser, G. F. Calvo, F. AgulloLopez, and M. Carrascosa, ibid. 65, 066610 (2002).

[7] S. Franke-Arnold, S. M. Barnett, M. J. Padgett, and L. Allen, Phys. Rev. A 65, 033823 (2002); H. H. Arnaut and G. A. Barbosa, Phys. Rev. Lett. 85, 286 (2000).

[8] Z. Chen, H. Martin, A. Bezryadina, D. Neshev, Y. S. Kivshar, and D. N. Christodoulides, J. Opt. Soc. Am. B 22, 1395
(2005).

[9] J. Yang and Z. H. Musslimani, Opt. Lett. 28, 2094 (2003); D. N. Neshev, T. J. Alexander, E. A. Ostrovskaya, Y. S. Kivshar, H. Martin, I. Makasyuk, and Z. Chen, Phys. Rev. Lett. 92, 123903 (2004); J. W. Fleischer, G. Bartal, O. Cohen, O. Manela, M. Segev, J. Hudock, and D. N. Christodoulides, ibid. 92, 123904 (2004); O. Manela, O. Cohen, G. Bartal, J. W. Fleischer, and M. Segev, Opt. Lett. 29, 2049 (2004); A. S. Desyatnikov, D. N. Neshev, Y. S. Kivshar, N. Sagemerten, D. Träger, J. Jägers, C. Denz, and Y. V. Kartashov, ibid. 30, 869 (2005).

[10] I. Makasyuk, Z. Chen, and J. Yang, Phys. Rev. Lett. 96, 223903 (2006).

[11] E. A. Ostrovskaya and Y. S. Kivshar, Phys. Rev. Lett. 90, 160407 (2003); 93, 160405 (2004).

[12] K. S. Buchanan, P. E. Roy, M. Grimsditch, F. Y. Fradin, K. Yu. Guslienko, S. D. Bader, and V. Novosad, Nat. Phys. 1, 172 (2005).

[13] I. V. Shadrivov and Y. S. Kivshar, J. Opt. A, Pure Appl. Opt. 7, 68 (2005). 
[14] In D. Jović, D. Arsenović, A. Strinić, M. Belić, and M. Petrović, Opt. Express 13, 4379 (2005), $L z$ for the backward beams is defined with a negative sign.

[15] M. Belić, M. Petrović, D. Jović, A. Strinić, D. Arsenović, K. Motzek, F. Kaiser, Ph. Jander, C. Denz, M. Tlidi, and Paul
Mandel, Opt. Express 12, 708 (2004).

[16] M. Belić, D. Jović, S. Prvanović, D. Arsenović, and M. Petrović, Opt. Express 14, 794 (2006).

[17] A. Ferrando, Phys. Rev. E 72, 036612 (2005). 